This is a self-archived version of an original article. This version may differ from the original in pagination and typographic details.

Author(s): Gerber, Nina; Booksmythe, Isobel; Kokko, Hanna

Title: Sex Allocation Theory for Facultatively Sexual Organisms Inhabiting Seasonal Environments : The Importance of Bet Hedging

Year: 2018

Version: Accepted version (Final draft)

Copyright: (c) 2018 by The University of Chicago.

Rights: In Copyright

Rights url: http://rightsstatements.org/page/InC/1.0/?language=en

Please cite the original version:

Gerber, N., Booksmythe, I., \& Kokko, H. (2018). Sex Allocation Theory for Facultatively Sexual Organisms Inhabiting Seasonal Environments : The Importance of Bet Hedging. American Naturalist, 192(2), 155-170. https://doi.org/10.1086/697727 


\title{
Sex Allocation Theory for Facultatively Sexual Organisms Inhabiting Seasonal Environments: The Importance of Bet Hedging
}

\author{
Nina Gerber, ${ }^{1,2,3, \star}$ Isobel Booksmythe, ${ }^{1,2}$ and Hanna Kokko ${ }^{1}$ \\ 1. Department of Evolutionary Biology and Environmental Studies, University of Zurich, Winterthurerstrasse 190, CH-8057 Zurich, \\ Switzerland; 2. Tvärminne Zoological Station, J.A. Palmenintie 260, 10900 Hanko, Finland; 3. Centre of Excellence in Biological \\ Interactions, Department of Biological and Environmental Science, University of Jyväskylä, Jyväskylä, Finland \\ Submitted November 2, 2017; Accepted February 20, 2018; Electronically published June 15, 2018 \\ Online enhancements: supplemental material, video. Dryad data: http://dx.doi.org/10.5061/dryad.885r162.
}

\begin{abstract}
AвSTRACT: Adaptive explanations for dormancy often invoke bet hedging, where reduced mean fitness can be adaptive if it associates with reduced fitness variance. Sex allocation theory typically ignores variance effects and focuses on mean fitness. For many cyclical parthenogens, these themes become linked, as only sexually produced eggs undergo the dormancy needed to survive harsh conditions. We ask how sex allocation and the timing of sex evolve when this constraint exists in the form of a trade-off between asexual reproduction and sexual production of dormant eggs - the former being crucial for within-season success and the latter for survival across seasons. We show that male production can be temporally separated from or co-occur with sex, depending on whether direct (time) or indirect (population density) cues of the season's end are available and whether population growth is density dependent. Sex generally occurs late in the season but is induced earlier in unpredictable environments. When only indirect cues are available, the temporal spread of sex, and with it the production of dormant stages, is even larger and, given sufficient mortality, leads to endogenous population cycles in which frequent sex coincides with high densities. In all scenarios, algorithms maximizing geometric mean fitness have reduced fitness variance compared with a hypothetical non-bet hedger, confirming that the timing of male production and sex in facultative seasonal settings can be bet-hedging traits.
\end{abstract}

Keywords: bet hedging, facultative sex, timing of sex, sex allocation, population density.

\section{Introduction}

Temporal variation in the environment leads to a major challenge: How do organisms adapt to often unpredictably fluctuating environments? If reliable cues are available, plasticity

\footnotetext{
* Corresponding author; email: nina.gerber@uzh.ch.

ORCIDs: Gerber, http://orcid.org/0000-0002-7142-1318; Booksmythe, http:// orcid.org/0000-0001-9108-1018

Correction: This article was reposted on June 22, 2018, to correct several errors in figure 2.
}

Am. Nat. 2018. Vol. 192, pp. 155-170. (C) 2018 by The University of Chicago. 0003-0147/2018/19202-58056\$15.00. All rights reserved.

DOI: $10.1086 / 697727$ can allow organisms to adjust their phenotype to current environmental conditions (Via and Lande 1985). However, organisms often cannot perfectly predict the demographic consequences of their current phenotypic choices. Finding the best life-history solutions can become challenging (McNamara et al. 2016), as simple maximization of mean fitness must be replaced with a more complicated set of trade-offs between mean fitness and its variance within and among individuals, which is summarized as bet-hedging theory (Slatkin 1974; review Starrfelt and Kokko 2012).

Dormancy is a classic example of a trait that has been suggested to evolve due to bet hedging (Evans and Dennehy 2005; Gourbière and Menu 2009). It has attracted much attention in plants (Ellner 1985; Gremer et al. 2012; Tielbörger et al. 2012; Gremer and Venable 2014) where germination probabilities are predicted to evolve to remain below 1 that is, not all seeds will germinate in the same season. Recent work considers analogous cases in animals like rotifers (García-Roger et al. 2014) and crustaceans (Pinceel et al. 2017), where eggs hatch after a variable delay. The general idea is that variable dormancy length can be beneficial if seasons vary in the suitability of conditions for the germinating (hatching) offspring; bet-hedging strategies buffer the risk of reproductive failure because not all offspring develop to experience the same environment (Cohen 1966).

In many organisms that engage in facultative sex (cyclical parthenogens), a mirror image of the plant germination case is encountered: the bet-hedging aspect of dormancy relates to its onset rather than its termination (though these can also coevolve; Spencer et al. 2001). In cyclical parthenogens, some generations are asexually produced and others sexually produced, a pattern that has evolved independently in several taxa, including Daphnia (Decaestecker et al. 2009), various insects (Simon et al. 2002; Burke et al. 2015), rotifers (Aparici et al. 1998), and many plants (Bengtsson and Ceplitis 2000). In such taxa, sexual reproduction is often tightly coupled with 
dormancy and/or dispersal (Simon et al. 2002; Ebert 2005; Schröder 2005), such that cost-benefit calculations of sex are driven overwhelmingly by the demographic effects that arise from the different fates of dormant and directly developing offspring (Stelzer and Lehtonen [2016] demonstrate this for rotifers). A parent has the choice of asexual reproduction, which contributes to immediate local population growth, or of adding to the pool of sexually produced dormant eggs that may also disperse.

Trade-offs between mean (arithmetic) fitness and its variance are particularly clear when the environment varies so much that some periods can only be survived in the dormant stage, and the timing of harsh conditions is difficult to predict. Asexual reproduction offers the potential for fast growth and competitiveness within a growing season, but sexual reproduction, which is the only means by which to produce dormant eggs, is ultimately necessary, or the entire lineage fails to survive to the next season, should dormancy be needed in between. Sex is thus crucial for long-term survival of a lineage, but when should it be employed?

When sexes are separate, an added complication is that three types of offspring may be produced. In Daphnia magna, on which we base our study, dormant eggs can be produced only sexually. A mother can produce either daughters or sons asexually or can mate with males and produce dormant eggs provided with a protective shell called an ephippium. Environmental cues can induce both male production and sexual reproduction and thus the formation of dormant ephippia. Day-length variation is an obvious cue of seasonal changes (Alekseev and Lampert 2001; Gilbert and Schröder 2004); for example, in northern Europe, shortening days that herald the approach of winter induce male production and sex (Roulin et al. 2013). However, hot spells in northern summers may also dry out populations in shallow water bodies. Additionally, if a population grows dense (via past asexuality), this can induce male production and sexual reproduction, for example, due to increased competition or reduced demographic cost of sex if high densities limit asexual growth (Gerber et al. 2018a). An unanswered question is whether such responses are shaped by bet hedging: Does a winning strategy achieve its success by modulating its variance in fitness in a beneficial direction, even if mean (arithmetic) fitness declines (Starrfelt and Kokko 2012), and does this depend on whether cues of the timing of the season's end are imprecise, indirect, or both? Our aim is thus to link sex allocation theory with the theory of bet hedging in a potentially complicated setting, where equal investment in male and female offspring is not the a priori expectation (because of generational overlap with seasonal variation [Kahn et al. 2015] combining with female reproduction not being constrained by mate availability when asexuality is an option [Booksmythe et al. 2018]).

Consider a strategy that begins reproducing sexually early in the season, largely forgoing the chance to fill the local en- vironment with asexually produced females. Within-season fitness is reduced, and at any point only a few mothers are available to produce ephippia (note that asexual Daphnia generations overlap within a season). However, early sex also has demographic benefits, as it reduces the variance in fitness; whether or not the season ends early, some ephippia have been produced.

Conversely, a strategy that begins sexual reproduction only late in the season may yield some fitness benefits: a long period of asexuality means that various demographic costs of sex (Lehtonen et al. 2012) are avoided for several generations. Once sex begins, the lineage comprises many mothers producing ephippia. As a consequence, a late-sex lineage is very productive if the season lasts sufficiently long. However, if the season ends sooner than sex was scheduled to begin, the entire lineage may still be asexual, with no ephippia produced to found the next generation. A risk-spreading strategy would then have been superior, producing at least some offspring equipped to survive the unfavorable season (Halkett et al. 2004; Tarazona et al. 2017).

While the above captures the essence of the problem, added complexity comes from the fact that sex requires males. In cyclical parthenogens, sex allocation decisionsthat is, offspring of which sex to produce - are not separate from which reproductive mode to invest in (sexual or asexual); production of males is pointless if no females are sexual in the near future. Currently, the only theoretical results for cyclical parthenogen sex allocation consider haplodiploid rotifers (Aparici et al. 1998; Serra et al. 2008). Their life cycles differ from our case as male abundance in rotifers directly affects whether mictic (sexual) females produce sons or daughters (fertilized eggs develop into females). Also, these studies have not considered stochastic variation in season length, so they do not comment on the relevance of bet hedging. Our model considers male production as part of the bet-hedging framework needed to understand the timing of sex in cyclical parthenogens.

\section{Methods}

The Daphnia System

In most Daphnia species, sexual reproduction is coupled with the formation of dormant eggs (for an exception, see Hebert 1981). Female Daphnia are iteroparous, with overlapping generations within a season, producing a clutch after every molt until death. Females can alternate between asexual and sexual reproduction throughout the season (fig. 1), producing three kinds of diploid offspring: asexually produced males and females and sexually produced dormant eggs that develop after dormancy into females. Note that in Daphnia sex determination is environmental, not genetic; thus, parthenogenetically produced offspring 


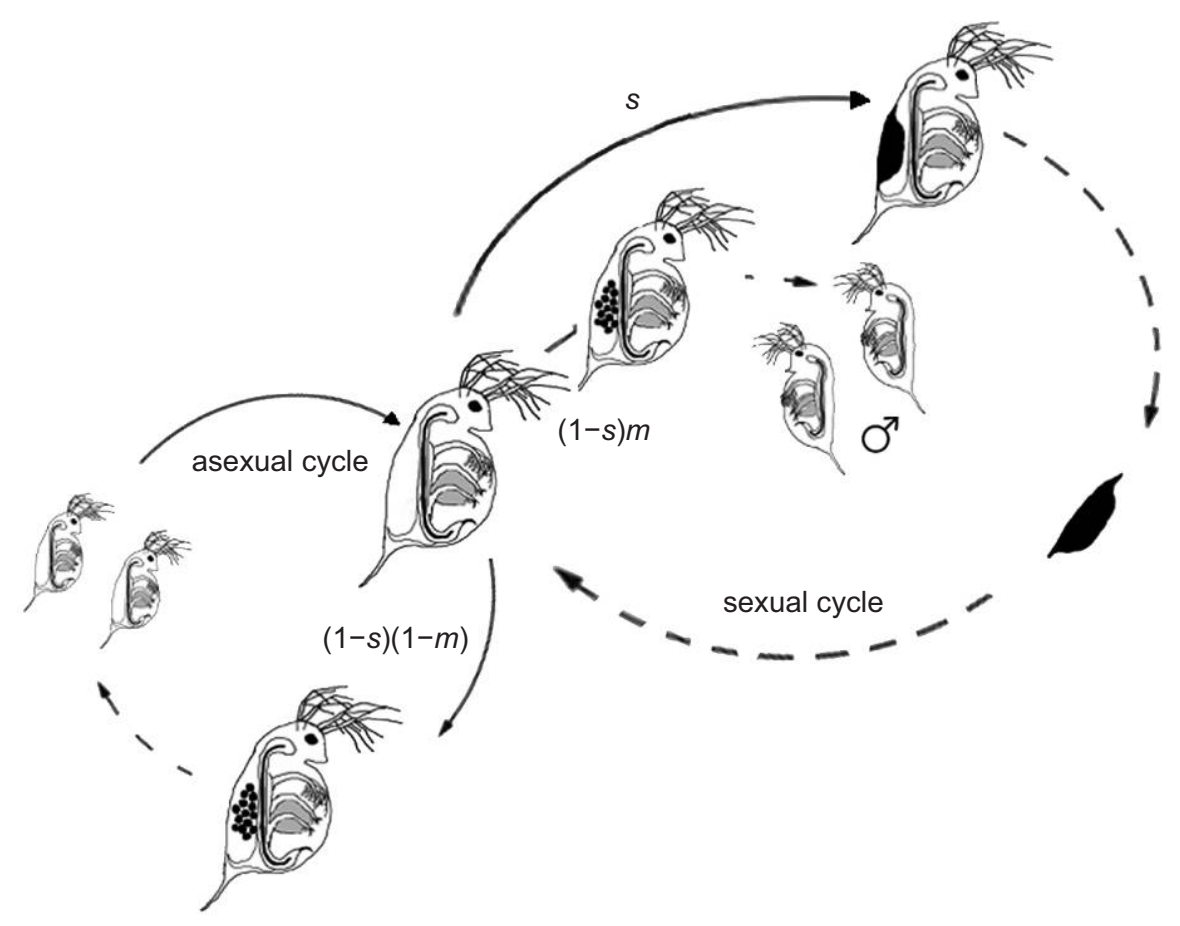

Figure 1: Daphnia life cycle. Female Daphnia have three reproductive options in every breeding cycle. A female can produce asexual daughters with probability $(1-s)(1-m)$, can produce asexual sons with probability $(1-s) m$, or can reproduce sexually and build an ephippium with probability $s$. The eggs that will be dormant inside an ephippium have to be fertilized by a male.

are clones of their mother, irrespective of their sex (Ebert 2005). Asexual clutch sex ratios are strongly biased toward one sex (Booksmythe et al. 2018). Therefore, we assume that a female produces either a clutch of females or a clutch of males in each asexual cycle.

The cost of sex is complicated to compute for cyclical parthenogens (Stelzer and Lehtonen 2016) but is clearly substantial within a season, as sex leads to no directly developing offspring. Producing males likewise trades off directly with production of daughters, who could increase the local density of females (each capable of eventual ephippia production). In addition, sexual reproduction in Daphnia entails a starkly reduced clutch size: females reproducing sexually produce only two eggs, while an asexual clutch can contain up to about 100 offspring (Ebert 2005; Gerber et al. 2018a). Finally, sexually produced eggs need to be fertilized by a male before being enclosed in the ephippium, which is released into the environment upon the female's next molt; a female committed to producing an ephippium might fail to get these eggs fertilized if no males were present.

\section{The Model}

The model is based on examining whether a mutant that uses a different schedule of male production and/or sexual reproduction from a resident strategy can spread in a vari- able environment. Thus, we aim to find a strategy that has reached such a state that alternative strategies cannot invade in the long term, when seasons vary in length. To find such a strategy, we need a measure for long-term fitness in an environment with variable season length that is based on the dynamics within each season. Here, we use a novel approach and extend the approach of geometric mean fitness by Starrfelt and Kokko (2012) with within-season calculations (fig. S1; video B1; figs. S1-S4 and video B1 are available online).

We first describe the fitness computations for each strategy. Thereafter, we describe how a genetic algorithm uses the relative fitness values of each strategy to update the best solution found so far and creates a new set of potential competitor values to be tested. This section will also justify the use of a genetic algorithm. One round of the genetic algorithm consists of evaluating the performance of several possible mutants, each of them evaluated with respect to all possible season lengths, against the currently best possible resident.

The fitness computations themselves represent a twostage process consisting of between- and within-season dynamics. First, we consider the between-season dynamics: What is the long-term fitness of a strategy with a known yearly sequence (distribution) of total ephippia produced? We use the approximation of geometric mean fitness (Starrfelt 
and Kokko 2012), justified when nonoverlapping ephippial generations follow each other in time. Note that this implicitly assumes that all ephippia begin contributing to populations in the season that immediately follows; this is realistic for Daphnia in shallow rock pools (Ebert 2005), where longterm deposits cannot accumulate. It is also a good approximation for other systems, as long as contributions from older sediments remain negligible. We next compute the withinseason dynamics of accumulated ephippia production for each strategy: What is the probability of a given strategy having produced $0,1, \ldots$, ephippia by the time the season ends? Our modeling tracks the accumulating ephippia production within a season for any given strategy. The realized accumulated number depends not only on the strategy but also on season length, modeled as a random variable.

We model fitness as a nonnegative real number; we do not force it to be an integer. This reflects our choice to focus on the environment (season length) as the dominant source of stochasticity in the system. This allows us to ignore demographic stochasticity, that is, the potentially nonidentical outcome of two runs of the same demographic process under an identical season length (because females with an identical propensity to produce ephippia or males might still differ in their realized choices in any one time step). Given our focus on environmental variation, we instead model the within-season dynamics deterministically (e.g., if the current probability of ephippia production is 0.3 and there are currently 1,000 females, they are recorded to yield an expected number of 300 ephippia in the current time step). The bet-hedging aspect arises irrespective of demographic stochasticity: fitness is unpredictable at the beginning of each season because the season length is not yet known.

Daphnia locally adapt to day length (Roulin et al. 2013), but reproductive decision-making can also be density dependent (Kleiven et al. 1992; Berg et al. 2001; Gerber et al. 2018a). We therefore make two alternative assumptions about how Daphnia infer time in the season: first, that they have perfect information about the time already elapsed (direct cue), and second, that this information is imperfect and the only cue available is current population density (indirect cue). Additionally, we consider two scenarios where population growth does or does not experience density dependence. The density-independent scenario is simpler (and might be accurate for a population that cannot saturate in a large water body), while the inclusion of density dependence is typically more realistic. We model the former (densityindependent growth) by assuming each asexual clutch leads to $c$ mature individuals, while in the latter case the number of maturing individuals declines with population density. (We denote the number of individuals that mature from one clutch as $c$, which captures the effect of clutch size as well as juvenile survival; see app. A, table A1 for model parameter definition). This yields a two-by-two table of assumptions

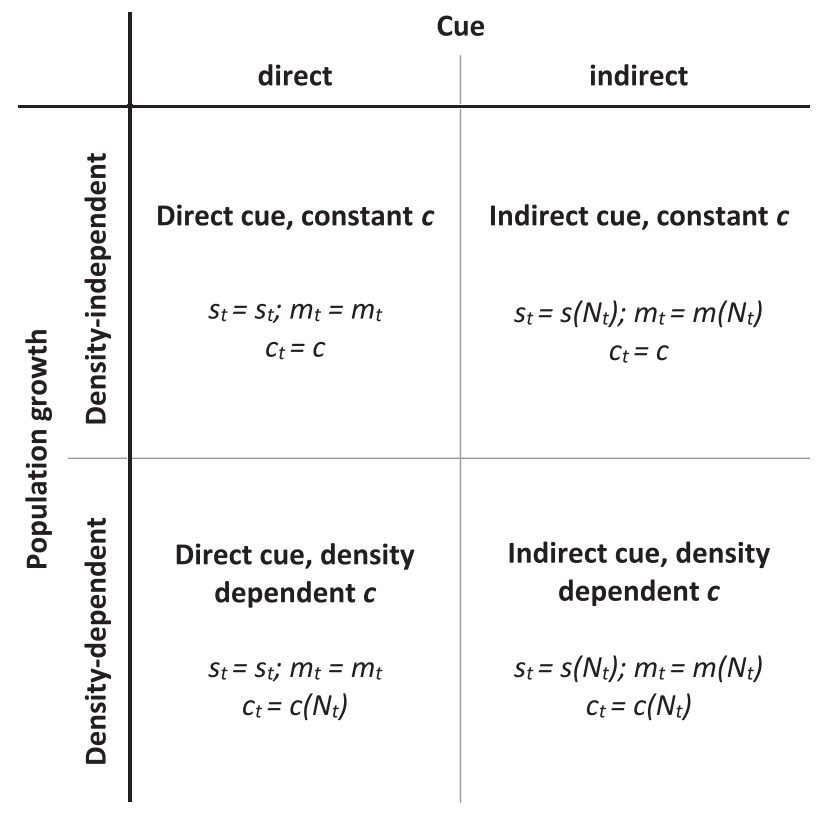

Figure 2: The two-by-two assumption sets.

such that density is allowed to impact the population as a cue only, as a factor influencing population growth only, as both, or as neither (fig. 2).

The definition of bet hedging is satisfied when a strategy reduces mean (arithmetic) fitness as well as the variance in fitness. Because reductions cannot be evaluated unless there is a baseline, we require a hypothetical non-bet hedger for comparison (Li et al. 2017). This hypothetical non-bet hedger obeys the same rules as above, but it maximizes arithmetic rather than geometric mean fitness. We present calculations based on this hypothetical organism to evaluate whether the timing of sex can be considered a bet-hedging trait.

The calculations below, wherever not specifically indicated to differ, are identical for each version of the model. The Matlab code to reproduce the model is deposited to Dryad (Gerber et al.2018b; http://dx.doi.org/10.5061/dryad.885r162) and is available in the supplemental materials as a zip file, available online. $^{1}$

Between-Season Dynamics. We base our model on the life history of Daphnia, confining our view of the benefits of sex to its strong demographic effect in this system: sex is required to survive harsh conditions because it is the only means by which to produce dormant eggs.

The probability that the season ends after $T$ breeding cycles is denoted $p_{T}$, and all active individuals die at this

1. Code that appears in The American Naturalist is provided as a convenience to the readers. It has not necessarily been tested as part of the peer review. 
point while ephippia produced during the season carry on their dormancy. The fitness of a strategy is defined by the geometric mean of ephippium production over all possible season lengths. We assume all ephippia hatch in the season following their production, irrespective of the precise timing of their production. A strategy's fitness $W$ is the geometric mean of the total number of gene copies, $G_{T}$, in dormant ephippia at the end of a season, computed for each possible season length $t$ and weighted by the probability that each season length occurs, $p_{T}$ (we assume that the maximum season length is $\left.\mathrm{T}_{\max }\right)$. Computationally, it is easier to work with the logarithm of fitness:

$$
\ln W=\sum_{T=T_{\min }}^{T_{\max }} p_{T} \ln G_{T} .
$$

Theoretically, the probability distribution $p_{T}$ can take any shape. We explore the effect of uniformly distributed probabilities, where $p_{T}$ takes the same value for all integers between $T_{\min }$ and $T_{\max }$ (we avoid setting $T_{\min }=1$, as seasons this short preclude the maturation of asexually produced young). Varying the difference between $T_{\min }$ and $T_{\max }$ allows us to explore different season predictabilities, from a highly predictable scenario where the season can end only at $T_{\max }$ to highly unpredictable scenarios where the shortest seasons, of length $T_{\min }$, are far shorter than the longest seasons that last $T_{\max }$ breeding cycles. We have chosen a uniform distribution to achieve a range of scenarios, from no variance in season length to very high variance. Note that the method of analysis could be applied to any desired shape (e.g., bimodal) of season-length distributions; it would involve simply entering a desired set of $p_{T}$ values in equation (1).

Within-Season Dynamics. The within-season dynamics specify what females do (using the options described in fig. 1) at each of the possible breeding cycles, from $t=1$ to $t=$ $T$ (the end of the season, which can go up to $T_{\max }$ or end sooner), and quantify the number of ephippia that accumulate up to the time point when the season ends. Asexual reproduction results in lineages sharing the same genotype, which defines the reproductive strategy. Genotypic similarity does not affect the strength of competition between clones. Different clones use strategies that differ in the probability of reproducing sexually at time $t$ (denoted $s_{t}$ ) and in the conditional probability of producing males if reproduction were not sexual (denoted $m_{t}$ ). Thus, at each time point a female's three options are an all-female clutch (probability $\left(1-s_{t}\right)$ $\left.\left(1-m_{t}\right)\right)$, an all-male clutch (probability $\left.\left(1-s_{t}\right) m_{t}\right)$, or an ephippium (probability $s_{t}$ ). The difference between the direct cue strategy set and indirect cue strategy set is that $s_{t}$ and $m_{t}$ are determined by time $t$ in the former but are a function of population density at time $t$ in the latter: $s_{t}=s\left(N_{t}\right)$ and $m_{t}=m\left(N_{t}\right)$.

Sexual clutch size is fixed to two eggs. We also need to account for the possibility that the number of gene copies present in these eggs, which is produced by a certain timing strategy, differs: if the male as well as the female are from the same clone, the genetic representation is doubled compared with outbreeding. Here we deviate from pure invasion analysis of a rare mutant, where mutants are assumed so rare that they do not encounter each other. Our rationale is that should the resident population produce no males, the ability of a mutant clone to reproduce sexually depends on its own male production, and we want to include this effect. Asexual clutch size varies and is typically much larger than two. Where population growth is density dependent, we assume a sigmoid relationship between $c$ and population density. This relationship is approximated from field data (Gerber et al. 2018a), with $c$ ranging from the mean asexual clutch size of the largest $10 \%$ of measured clutches at low densities to $\sim 0$ at very high densities (fig. S2). Where population growth is density independent, we keep $c$ constant. When carrying capacity is reached, individuals can still reproduce sexually because sexually produced eggs do not contribute to current population density, and empirical data show that high densities do not impede the production of sexual clutches (Gerber et al. 2018a). We assume equal survival for male and female clutches.

We follow the dynamics of a rare mutant clone in a population otherwise consisting of a resident strategy. Mutants differ from residents only with respect to timing; thus, our model operates in the absence of any other differences in competitive ability. Below, we use $s_{t}$ and $m_{t}$ to refer to the strategy set regardless of whether cues are direct (time) or indirect (population density); the difference in interpretation is that if population densities are the same at two time points, the corresponding $s_{t}$ and $m_{t}$ values may differ in the direct cue setting but must be the same if cues are indirect. We use the superscript $r$ when referring to the resident strategy, contrasting with the mutant strategy (denoted by ${ }^{\prime}$ ) whose fitness we evaluate.

We first calculate the dynamics of the resident population strategy that determines the environment in which a mutant strategy's fitness is evaluated. Each female following the resident strategy reproduces sexually with probability $s_{t}^{r}$ at time $t$ and produces males with probability $m_{t}^{r}$ if reproducing asexually. To compute the density of mature individuals in the resident population at time $t$, we consider that asexually produced males and females require two breeding cycles to reach maturity. The mortality of mature individuals is assumed to equal $d_{\mathrm{f}}$ (females) or $d_{\mathrm{m}}$ (males) when measured over one breeding cycle.

The number of mature females at time $t\left(F_{t}^{r}\right)$ equals the newly matured females that were produced at time 
$t-2$, plus the number of surviving previously mature females $\left(F_{t-1}^{r}\right)$ :

$$
F_{t}^{r}=F_{t-2}^{r} c\left(1-s_{t-2}^{r}\right)\left(1-m_{t-2}^{r}\right)+F_{t-1}^{r}\left(1-d_{\mathrm{f}}\right) .
$$

The equivalent male equation reads

$$
M_{t}^{r}=F_{t-2}^{r} c\left(1-s_{t-2}^{r}\right) m_{t-2}^{r}+M_{t-1}^{r}\left(1-d_{\mathrm{m}}\right) .
$$

The sequence of $F_{t}^{r}, M_{t}^{r}$ values forms the environment in which a mutant's fitness is evaluated. Mutant dynamics $\left(F_{t}^{\prime}\right.$ and $\left.M_{t}^{\prime}\right)$ are computed analogously to equations (2a) and (2b),

$$
\begin{aligned}
F_{t}^{\prime} & =F_{t-2}^{\prime} c\left(1-s_{t-2}^{\prime}\right)\left(1-m_{t-2}^{\prime}\right)+F_{t-1}^{\prime}\left(1-d_{\mathrm{f}}\right), \\
M_{t}^{\prime} & =F_{t-2}^{\prime} c\left(1-s_{t-2}^{\prime}\right) m_{t-2}^{\prime}+M_{t-1}^{\prime}\left(1-d_{\mathrm{m}}\right) .
\end{aligned}
$$

To compute mutant fitness, we assume the population starts with one mutant among a much larger number of resident females (999 in our examples, bringing the total population to 1,000; note that in Daphnia, all individuals hatching from ephippia are female). In practice, the ratio of mutants to residents matters little to the outcome.

Sexual reproduction requires that males are present in the population. Whenever $M_{t}^{r}+M_{t}^{\prime}>0$, the total number of ephippia produced at time $t$ by the mutant clone equals $F_{t}^{\prime} s_{t}^{\prime}$. Each ephippium contributes, on average, fitness $1+M_{t}^{\prime}$ / $\left(M_{t}^{r}+M_{t}^{\prime}\right)$ to the clone that produced it $\left(M_{t}^{\prime} /\left(M_{t}^{r}+M_{t}^{\prime}\right)\right.$ being the probability that the father belongs to the same clone); simultaneously, the resident population produces $F_{t}^{r} s_{t}^{r}$ ephippia, and each of these contributes, on average, $M_{t}^{\prime} /\left(M_{t}^{r}+\right.$ $\left.M_{t}^{\prime}\right)$ units of fitness to the mutant clone via paternity. Thus, the fitness accrued by the mutant strategy at time $t$ is

$g_{t}^{\prime}=\left\{\begin{array}{cl}F_{t}^{\prime} s_{t}^{\prime}\left(1+M_{t}^{\prime} /\left(M_{t}^{r}+M_{t}^{\prime}\right)\right)+F_{t}^{r} s_{t}^{r} M_{t}^{\prime} /\left(M_{t}^{r}+M_{t}^{\prime}\right) & \text { if } M_{t}^{r}+M_{t}^{\prime}>0, \\ 0 & \text { otherwise. }\end{array}\right.$

If the season lasts until $t=T$, total fitness accumulation equals

$$
G_{T}=\sum_{t=1}^{T} g_{t} .
$$

The value $G_{T}$ is inserted into the between-season dynamics equation (eq. [1]). A full evaluation of the value of equation (1) requires as many calculations of equation (5) as there are possible season lengths, $T$. The resulting $G_{T}$ for each possible $T$ is then weighted with the probability $p_{T}$ that this length occurs in nature (eq. [1]). Note that this approach does not involve replacing the resident with a mutant as soon as the latter has a superior $G_{T}$ value in any specific season, which could erroneously lead to assigning high success to a strategy with no long-term prospects. Instead, the winner (based on eq. [1]) is determined only once success has been computed over the entire distribution of season lengths that can occur in nature.
Genetic Algorithm. In principle, the above steps are sufficient to find an overall winner. Practically, however, finding the fitness-maximizing values for $s$ and $m$ for every time point $t$ is challenging, for several reasons. First, while $s$ and $m$ can have independently evolving values at every breeding cycle or population density (depending on model version), this may conflict with the general notion that we might not expect perfectly fine-tuned evolutionary responses to minor variations in the environment (McNamara et al. 1997; McNamara and Houston 2009). Second, the chosen strategy at time $t$ can influence future time points (e.g., a diminishing proportion of males produced at time $t$ are still available in future breeding cycles). Third, the optimal strategy depends not only on the mutant clone's behavior but also on the behavior of the resident population; for example, male production pays off less well if there are no outbreeding opportunities because the resident population produces no ephippia. On the other hand, sex is possible without producing males if others produce them but requires own male production if not. Finally, we must calculate the geometric mean as a fitness measure for every possible season end. Based on the complexity of the situation, we do not seek analytical solutions but use a genetic algorithm that follows haploid sexual reproduction. Note that we choose a genetic algorithm not to accurately simulate chromosomes of Daphnia but as a tool to find solutions to a complex life-history problem.

The Algorithm. First, we create a resident strategy $s_{t}^{r}$ and $m_{t}^{r}$ for every breeding cycle by drawing uniformly distributed random numbers between 0 and 1 for every $t$. This is simple for the direct cue scenario, but it requires that $s_{t}^{r}$ obeys a functional form $s^{r}\left(N_{t}\right)$ in the indirect cue scenario. For the latter we group the logarithmic population density into 100 categorical bins with logarithmically spaced borders, such that the first bin contains all log densities between 0 and 1 , the second bin between 1 and $2, \ldots$, until 100 (the largest population density our model can handle is $e^{100}$, which is sufficiently large to never be reached). The value $s(N)$ is assumed equal for the range of population densities in one bin. In the next step, nine mutant strategies $s_{t}^{\prime}$ and $m_{t}^{\prime}\left(\right.$ or $s^{\prime}\left(N_{t}\right)$ and $\left.m^{\prime}\left(N_{t}\right)\right)$ are created by adding normally distributed random numbers to the resident strategy. Values below 0 are set to 0 and values larger than 1 are set to 1 , so that $1>s_{\text {bin }}^{\prime}, m_{\text {bin }}^{\prime}, s_{t}^{\prime}, m_{t}^{\prime}>0$.

Using equations (2) and (3), we can calculate the dynamics of the resident and mutant strategies and the fitness of each strategy; this is given by equation (1), except for the case of a hypothetical non-bet hedger, for which equation (1) is replaced by

$$
W=\frac{1}{T_{\max }} \sum_{T=1}^{T_{\max }} p_{T} G_{T} .
$$


We thereafter keep the two strategies that yield the highest fitness and the resident strategy. These three strategies offer three options for $s$ and $m$ for each breeding cycle (or density bin). The algorithm creates nine recombinant strategies by randomly choosing, for each breeding cycle (or density bin), a value with a probability of $1 / 3$ from any of the three options available. Additionally, with probability $p$ (independently applied for each cycle or bin of each recombinant strategy), the recombinant may take its value from a temporally adjacent breeding cycle (or density bin). This speeds up the optimization process because well-performing actions at time $T$ can spread to temporally neighboring points. Strategies at the first and last breeding cycle (or density bin) have only one adjacent strategy from which to copy.

Finally, the fitness of the original resident and the recombinant strategies are calculated as described for the mutant strategies. The strategy with the highest fitness becomes the new resident strategy from which mutants are created in the next round of the algorithm.

This algorithm optimizes the values for $s_{t}$ and $m_{t}$ at every breeding cycle within a season. Assuming convergence, this yields the approximately optimal strategy of the timing of sexual reproduction and male production. For each scenario we ran the algorithm until the optimization criterion (eq. [6]) remained unchanged for 100 iterations. This criterion was reached after a mean of 6,238.8 to a mean of 10,358 rounds, depending on scenario. Instead of averaging independent runs, we present the results of the best-performing strategy of 10 independent runs, since we are able to compare in absolute terms the performance of strategies produced by a genetic algorithm and because finding a better one makes a previously found, less well-performing strategy obsolete.

\section{Results \\ Direct Cue of Time: Density-Independent Population Growth}

Situations in which females have information on how far the season has progressed, and population growth is density independent, present the simplest case of all our model versions. If we additionally assume that the season end is perfectly predictable (fig. 3, top), females only invest in sexual reproduction very late: it is more efficient to reproduce asexually unless the season end is imminent. To be precise, females switch to sexual reproduction two breeding cycles before the season ends (fig. 3). Because we assume it takes two breeding cycles for directly developing offspring to mature, it is optimal to switch to sex when asexually produced daughters no longer have time to mature and contribute to reproduction. Similarly, because males also need two breeding cycles to mature, and successful sex requires males, they are produced two breeding cycles before sex is induced (fig. 3, top). Predictable conditions therefore lead to late sex, and the situation additionally recovers, at least to an approximate degree, the familiar principle of equal investment in sex allocation (West 2009): during male production (time steps 27 and 28 in fig. 3 , top), the sex ratio of newly produced offspring deviates only slightly from $50 \%$. The deviation is in the direction of more male production $(>50 \%)$, and it is easy to understand: any male produced arises during these time steps, while females may have been produced earlier, and some of these females may still be alive when sexual reproduction commences.

Increasing uncertainty leads to a far wider spread of sex and male production (fig. 3, middle and bottom) and to some inaccuracy of the best algorithm, as a small fraction of males are produced later than they can possibly mature (fig. 3 , middle and bottom, at times 29 and 30, respectively; note that very few generations actually experience seasons this long, which helps to explain the approximate performance of the algorithm at this end). Any straightforward expectation of equal investment becomes unjustified, a result known from sex allocation theory when scenarios involve overlapping generations and sequential decisions with mortality occurring in between (West 2009; Kahn et al. 2015). As an additional complication, the facultative nature of sex means that males can hope to fertilize eggs only when females commit to sexual egg production; thus, we expect deviations from $1: 1$ sex ratios mostly in the downward (fewer males) direction.

The model confirms this expectation and predicts that uncertain season lengths make the production of males and sexual eggs overlap, with an increase that is shallower than the cumulative probability that the season has ended by time $t$. The common feature between all cases is that male production commences precisely two breeding cycles before the production of sexual eggs, reflecting our assumption of a fixed time to maturity. While conceptually clear, this densityindependent model scenario is unrealistic for long seasons, as populations reach unrealistically high densities $\left(10^{17}-10^{19}\right.$ individuals).

\section{Direct Cue of Time: Density-Dependent Population Growth}

Introducing density dependence retains many of the patterns of the density-independent model while keeping population densities within a realistic range. Male production precedes sexual reproduction, and increased unpredictability of the season length broadens the time over which sex occurs. The main difference from the density-independent model is that asexual female production ceases as soon as carrying capacity is reached (fig. 4). If adult mortality is low this cessation may be permanent, and adult population size gradually declines over the remainder of the season (fig. 4, middle and bottom) or, in the predictable-season-end case, even earlier (fig. 4, 

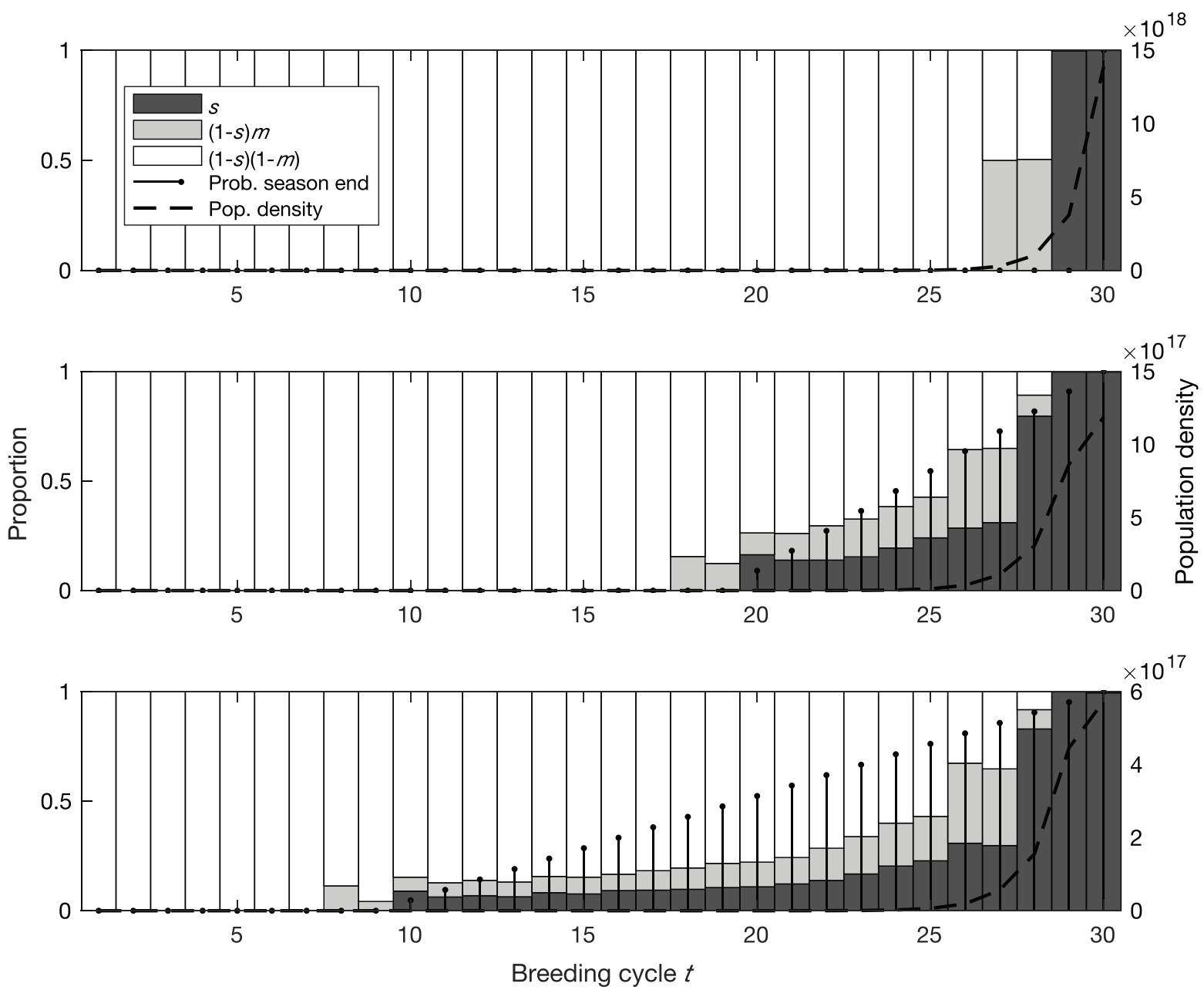

Figure 3: Direct cue, density-independent population growth. The optimal proportions of sexual reproduction, $s$, and male production $(1-s) m$, at every breeding cycle in a season following the assumptions in the lower left-hand corner of figure 2. The remaining white area therefore indicates female production, $(1-s)(1-m)$. Panels illustrate three different predictabilities of the season end (the cumulative probability of which is indicated with vertical lines); we show the best strategy of 10 independent runs of the genetic algorithm. Panels show that (top) the season will predictably end at $T_{\min }=T_{\max }=30$; (middle) there are 11 possible end times between $T_{\min }=20$ and $T_{\max }=30$; and (bottom) there are 21 possible end times between $T_{\min }=10$ and $T_{\max }=30$. All panels use $d_{\mathrm{f}}=d_{\mathrm{m}}=0.05, c=10$.

top). During this time, existing females simply release ephippia; because males die at the same rate as females, they are mostly still available to fertilize the ephippial eggs.

The above assumes low mortality, such that the adult population size decays slowly in the absence of new, directly developing offspring. If adult mortality increases and the season length is very unpredictable (fig. S3), the solution changes to an early peak of sexual reproduction (preceded by male production) during which female production does not completely cease, followed by a switch back to the predominant production of directly developing females, which in turn precedes the final return to sexual reproduction (again with male production first). In this setting, by the time at which it is possible but unlikely that the season has ended, some ephippia will have been produced, while most sex hap- pens later, when the cumulative probability of the season having ended becomes appreciable. The population density shows, correspondingly, two peaks (fig. S3c).

\section{Indirect Cue (Density Is a Cue for Time): Density-Independent Growth}

There is little relative difference between populations with access to direct cues (fig. 3) and those that rely on the indirect cue of population density (fig. 5). However, the latter case shows mild signs of the algorithm having produced only an approximate solution: in figure 5, top, male production begins three breeding cycles before eggs requiring fertilization appear. This may reflect computational inaccuracies as we were 

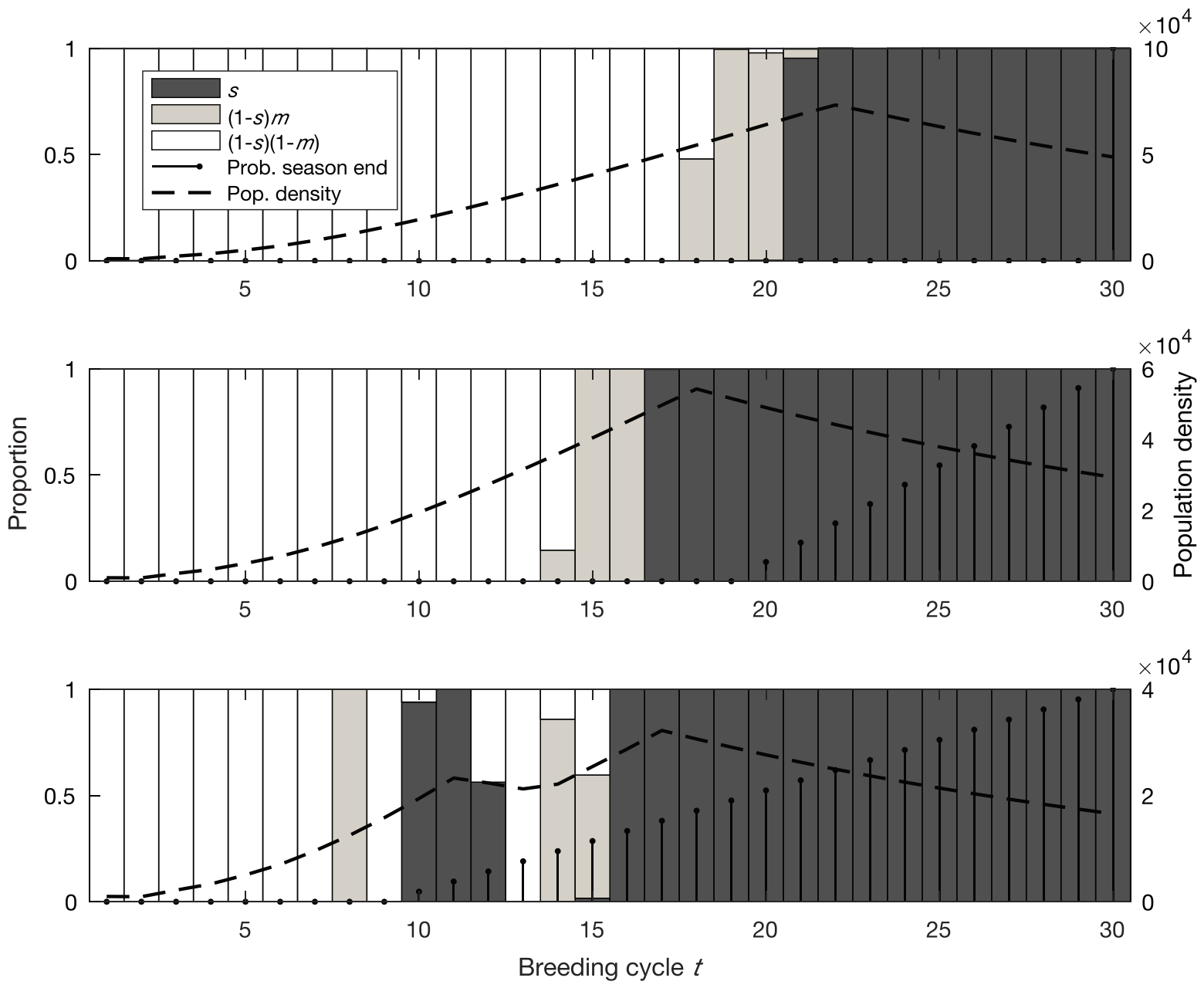

Figure 4: Direct cue, density-dependent population growth. The same notation and parameter values as in figure 3, with assumptions following the top left-hand corner in figure 2 , where recruitment $c$ obeys the function $\exp (4+(-5-4) /(1+\exp ((-0.3)(\ln (\operatorname{density})-8))))$ (see fig. S2).

forced to assume identical responses to all within-bin population densities that occurred in a population.

\section{Indirect Cue (Density Is a Cue for Time): Density-Dependent Population Growth}

When population growth is density dependent and density itself acts as a cue to switch between reproductive modes, we find qualitatively the same pattern as before: densitydependent population growth greatly broadens the time over which sex occurs (fig. 6) compared with the densityindependent scenario (fig. 5). If adult mortality is low, the asexual production of directly developing females is low over much of the season (fig. 6). Higher adult mortality creates a stronger need to replenish the population of adult females should the season continue (fig. S4), and the details of season length then determine whether population density shows cyclic behavior or a slow increase or decline (fig. S4). At high- and low-season-length predictabilities, male and sexual egg production overlap, and population density stays relatively constant (figs. 6, top, bottom, S4a, S4c). Interestingly, intermediate-season-length predictability can produce density cycles, where peaks correspond with the highest prevalence of sex (figs. 6, middle, S4b). Frequent sex leads to a decline in population size (adult mortality combines with little asexual offspring production), which in turn induces asexual production of males and females. The sex allocation pattern during this phase shows fewer males if adult mortality is higher (contrast fig. S $4 b$ with fig. 6, middle).

\section{Evaluation of Bet Hedging}

To evaluate whether a strategy selected to optimize geometric mean fitness (measured as total ephippia produc- 

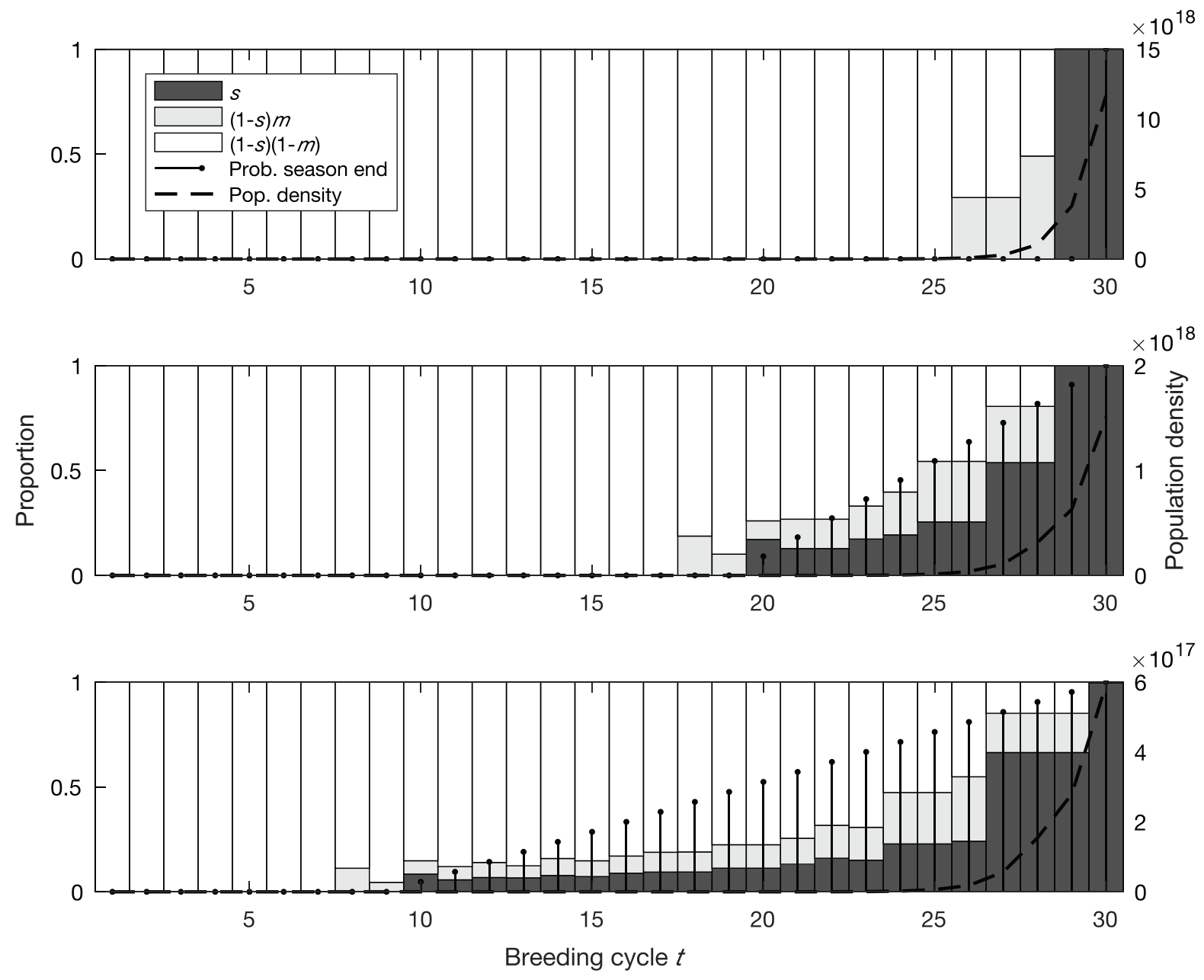

Figure 5: Indirect cue, density-independent population growth. The same notation and parameter values as in figure 3, but with assumptions following the top right-hand corner of figure 2 .

tion over multiple seasons) meets criteria for bet hedging, we compared the arithmetic mean and variance in ephippia produced by this strategy with the mean and variance produced by a hypothetical non-bet hedger selected to optimize arithmetic mean fitness. Although this non-bet hedger cannot persist in nature if it produces zero offspring in any given season, it forms the necessary baseline that allows us to evaluate the fitness moments that helped the evolved strategy express its demographic superiority. Relative to the nonbet hedger, we expect a decrease in both the arithmetic mean fitness and variance in fitness of the strategy optimizing geometric mean fitness, if this strategy is a bet hedger.

Absolute fitness means and variances differed greatly across model assumption sets (fig. 7); however, bet-hedging criteria were satisfied in all environments (which generally spanned a larger range of potential season lengths than the examples shown in figs. 3-6). The difference in variance was not always of the same magnitude as the difference in mean (lines in fig. 7 are not parallel), and the absolute magnitude of the differences depended strongly on the unpredictability of the environment. As expected, a shorter period of uncertainty (a late $T_{\min }$ ) led to a smaller difference in either fitness moment between the bet hedger and nonbet hedger.

\section{Discussion}

Strategies of sex allocation and reproductive mode in cyclical parthenogens are poorly understood, especially when environments are unpredictable. Our models show that for facultatively sexual organisms, spreading the use of sexual reproduction, and with this the production of dormant eggs, over several breeding cycles can be adaptive in unpredictable environments, and this strategy satisfies criteria for 

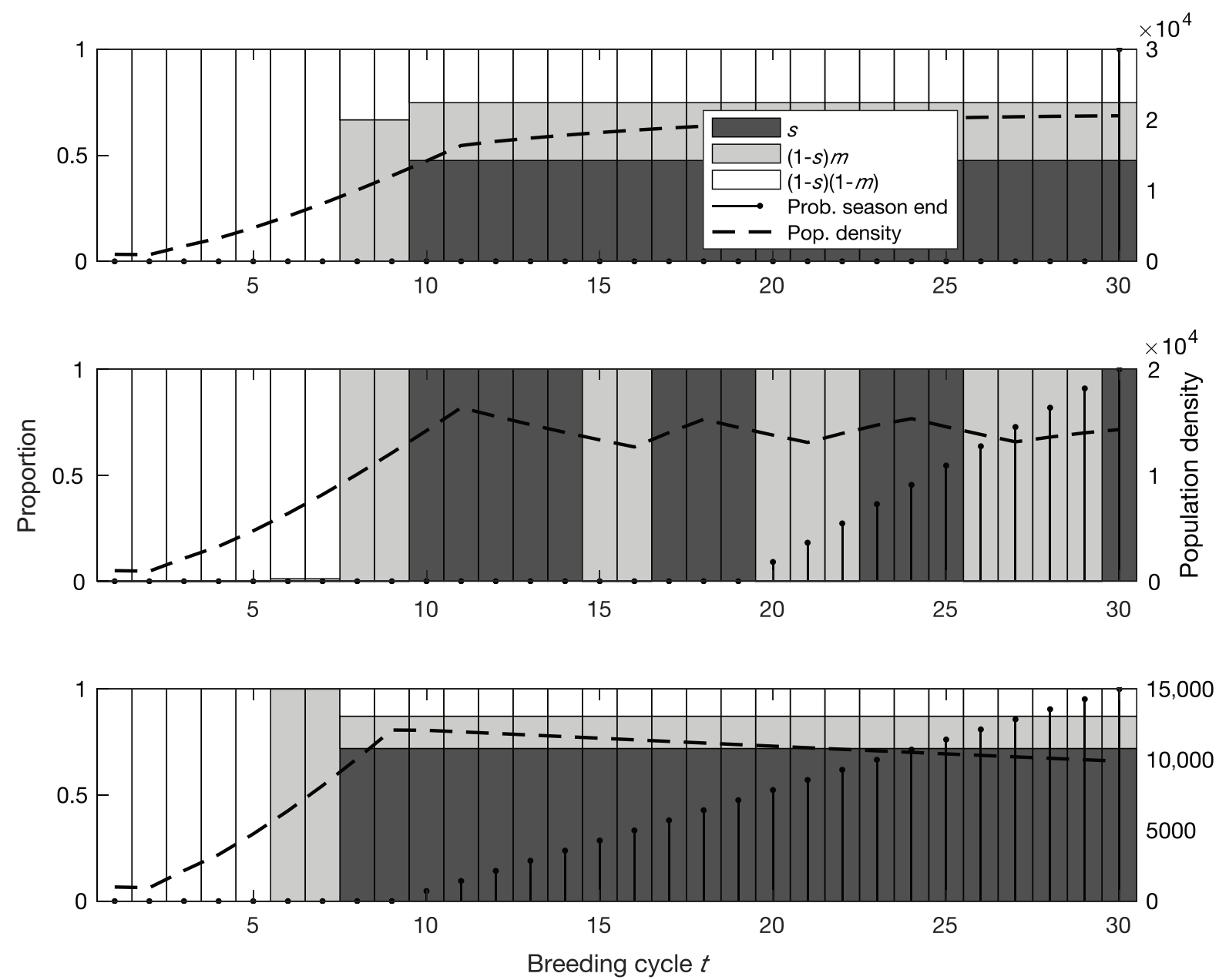

Figure 6: Indirect cue, density-dependent population growth. The same notation and parameter values as figure 3, with assumptions following the lower right-hand corner in figure 2, and thus assumptions regarding recruitment $c$ follow those of figure 4 .

bet hedging. When season length is unpredictable, the induction of sexual reproduction is driven by the temporal probability distribution of the length of the season, with important modifications that depend both on the seasonal cues available and whether recruitment is density dependent.

When the end of the season is predictable, sexual production of dormant eggs only occurs shortly before the season actually ends. In all other environments, sexual production of dormant eggs is a risk-spreading strategy, with some ephippia produced as soon as there is some risk that the season might end and male production beginning strategically as long beforehand as is needed for the first male generation to mature. Our finding that sex is spread more widely when season length is more variable is consistent with models based on other cyclical parthenogen life histories, such as those of aphids, where optimal investment in sexual reproduction is proportional to the variance in the season end
(Halkett et al. 2004). A gradual and precocious induction of sex in uncertain environments was also described in a general model of cyclical parthenogens, where sexual reproduction is linked with dormancy (Spencer et al. 2001).

These earlier studies, however, did not link their findings with literature on sex allocation. In this context, cyclical parthenogens present another set of problems (Aparici et al. 1998; Serra et al. 2008). For example, is there a sense in which equal allocation to males and females is maintained? In some qualified sense the answer may be yes; in monogonont rotifers, sexual females are a specific subset of all females, and male production may evolve to match their number (Serra et al. 2008). However, when a specific female does not have to commit to sex (e.g., in Daphnia, females can switch back and forth between reproductive modes), it is less clear whether one can expect any general insight regarding total investment. This is due to the dynamic nature of sex allocation, where males produced in different 

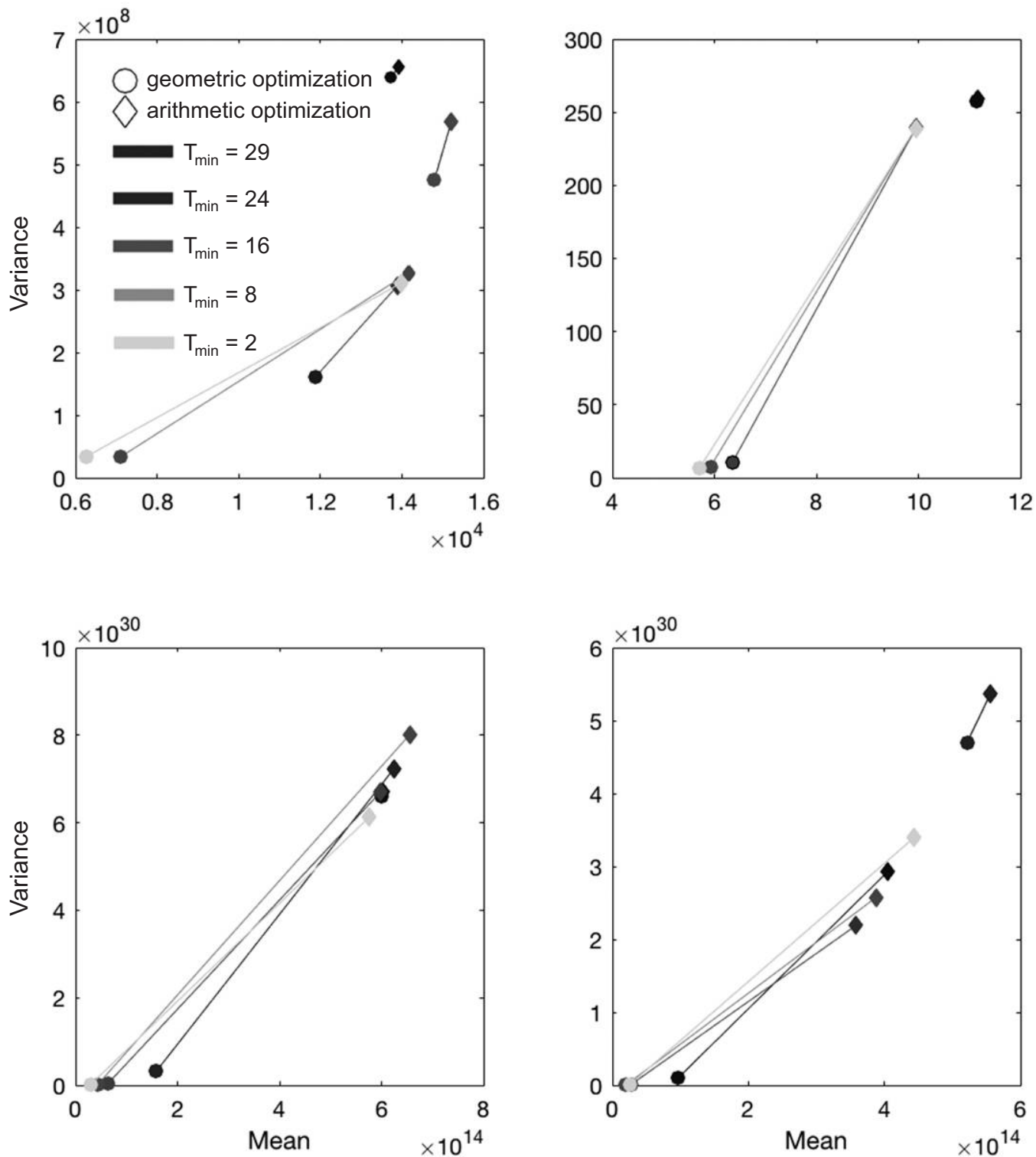

Figure 7: Bet hedging. Comparison of the (arithmetic) mean and variance in ephippia production for strategies selected to optimize either the arithmetic mean (diamond) or the geometric mean (circle) ephippia production. The four tested scenarios correspond with those listed in figure 2. All panels use $d_{\mathrm{f}}=d_{\mathrm{m}}=0.05$ and $T_{\max }=30$, with recruitment $c$ being either fixed at 10 (top row) or density dependent (bottom row) as described in figure S2.

time steps may coexist and compete for females - only some of which offer fertilization opportunities (Booksmythe et al. 2018). In alignment with other situations in which a sequential nature of interactions (including mortality) can blur an expectation based on investments (Kahn et al. 2015), our results highlight that near-equal sex ratios are produced only in rather exceptional circumstances, where all females are about to turn sexual very soon.

A more general question, then, arises: Should male production take place mainly before sexual reproduction begins, or should it co-occur with sex to enable replenishment of the male population? We find the former solution more often if individuals have direct cues of how late it is in the season, allowing for more precisely orchestrated timing of each category of individuals. The alternative, where some females produce male clutches while others produce females and yet others develop eggs requiring fertilization, tends to associate with indirect cues, although not categorically. Indirect cues can also lead to temporal separation of male production and sex, if at high density sex becomes so prevalent that the current pop- 
ulation declines. Once it has decreased below a threshold, the best strategy switches to replenishing the adult population, and male production once again anticipates future mating opportunities (Kahn et al. 2013).

How well do our predictions match what is found in nature? In predictable habitats we show that sexual formation of dormant eggs should be delayed, with a peak toward the predetermined season end or at high population densities. Empirical studies find that Daphnia populations in predictable habitats, such as permanent water bodies in seasonal, temperate environments, produce ephippia only at the end of the season (Galimov et al. 2011), whereas Daphnia from shallow ponds at northern latitudes, which freeze over winter and occasionally dry out in summer, produce ephippia throughout the season (Altermatt and Ebert 2008; Roulin et al. 2013; Gerber et al. 2018a). While studies that consider the timing of male presence in a population tend to focus on the co-occurrence of males with sexually reproducing females, rather than the timing of male production per se, many of these detect males substantially earlier than the appearance of ephippial females (e.g., Galimov et al. 2011). Our own empirical results (Gerber et al. 2018a), as well as those of a previous study (Innes 1997), track clutch sex ratios over time in natural Daphnia populations and additionally show that male production continues throughout the season, overlapping with ephippia production.

Likewise, our results on density appear well supported by data. When we included density-dependent recruitment in the model, sex was induced as soon as population density peaked, in some cases showing multiple peaks. One interpretation is that the direct demographic cost of sex, relative to asexual reproduction, is reduced at high population densities because asexuality becomes inefficient. In several cyclical parthenogens, population density is an important predictor of the frequency of sex (Daphnia: Carvalho and Hughes 1983; Kleiven et al. 1992; Gerber et al. 2018a; rotifers: Serra et al. 2008; Stelzer and Snell 2003). In our model, providing the algorithm with information on population density was sufficient to adapt the propensity for sex to the temporal pattern of season length, in the absence of direct information about the season's progression.

For density cues to work in a bet-hedging framework, this requires a correlation between time (since the beginning of the season) and population density. In seasonal populations with few founders at the beginning of each season and a large population later on, such a correlation arises fairly automatically. Interestingly, with sufficient adult mortality, our model showed the possibility of endogenous population cycles: mortality exceeded recruitment during high density (and consequent high sex) periods, which were followed by periods of lower density, which in turn led back to asexuality and an increase in short-term population growth. Determining whether observed cycles in nature result from this process requires more fine-scaled analysis than the mere observation that sex peaks at high density, although this observation is consistent with the above reasoning. High rates of sex necessarily lead to lower population growth when sexual offspring cannot recruit and mature in the near future (Stelzer 2012), so the demographic features of our model should apply to many systems. More details would be welcome from natural systems; for instance, we did not allow individuals using indirect cues to perceive the density of males and females separately, even though this may be possible for Daphnia (Booksmythe et al. 2018).

Regardless of the details of our model variants, we found the timing of sex in cyclical parthenogens to represent a bethedging strategy, where mean fitness is traded off against a reduction in fitness variance. Empirical studies have also shown that the formation of dormant stages can represent bet hedging when the season is unpredictable or cues are uncertain (Bradford and Roff 1993; Graham et al. 2014; Furness et al. 2015). It is interesting to reflect on the classic examples of bet hedging that consider the optimal duration of dormancy in plants (Cohen 1966; Ellner 1985) or animals (Hanski 1988). Incorporating the possibility of additional bet hedging in ephippial dormancy duration might influence the optimal strategy for sex induction in our model, because failing to produce ephippia in one season does not guarantee lineage extinction when ephippia from past seasons can hatch in the future. However, our model should provide a good approximation for systems where long-term accumulation of dormant stages is impossible or when reaching hatching conditions again becomes highly unlikely, due, for example, to habitat structure or high predation risk. Earlier theoretical work investigating the interaction of the timing of sex with the hatching fraction of eggs (Spencer et al. 2001) shows low hatching (interpretable as high bet hedging) covaries with situations that lead to high variability in within-season reproductive output. Thus, the need to hedge bets within a season might not readily disappear even if organisms invest in another bet-hedging strategy (delayed hatching).

When sexual reproduction is linked with an ecological function (such as dormancy), which is crucial for survival through some range of the temporal variation in an environment, cyclical parthenogenesis is always superior to a pure asexual strategy. This upholds the best-of-both-worlds view of the benefits of facultative sex: periods of fast demographic growth (avoiding costs of sex) alternate with periods of investment in sex (as a long-term survival strategy). Often, facultative sex is said to offer the best of both worlds in a different setting: reaping the genetic benefits of sex while (in most generations) avoiding its costs (D'Souza and Michiels 2010; Burke and Bonduriansky 2017). Our model focuses on the marked differences in the demographic consequences of sexual and asexual reproduction, which exist whenever sex and dormancy are linked, and does not consider the potential 
genetic benefits of sex. Although genetic consequences of sex are known to scale nonlinearly with the frequency of sex (Green and Noakes 1995), accounting for this would probably not change the timing of sex much in our model because all founding individuals at the season's beginning are produced sexually, and offspring produced thereafter are their clones (leaving little scope for direct competition between sexual and asexual genotypes, which could be affected by processes considered by Green and Noakes [1995]).

Our model rests on the premise that sex and dormancy are tightly coupled, an assumption we base on findings that this is indeed widespread in organisms as diverse as Cladocerans (Tessier and Caceres 2004; Wittmann et al. 2011), aphids (Simon et al. 2002), rotifers (Carmona et al. 2009), and oligochaete worms (Schierwater and Hauenschild 1990) as well as many plants, where resistant seeds are sexually produced. While our model (and that of Spencer et al. 2001) is silent on why this link exists, an obvious next question is whether there is an adaptive reason to expect dormant forms to be sexually produced, while directly developing offspring result from parthenogenesis - especially because some exceptions exist (for an obligately asexual Daphnia lineage that can produce dormant stages asexually, see Innes et al. 2000; bdelloid rotifers behave similarly; see Caprioli and Ricci 2001). We address this question by relaxing the assumption of a preexisting constraint (Gerber and Kokko 2018).

In conclusion, we show that spreading sexual reproduction over several breeding cycles within a season is expected to evolve in unpredictable environments such that mean arithmetic fitness is traded off against variance in offspring survival (via dormancy) across seasons. This confirms that bet hedging between dormant versus directly developing offspring represents an important mechanism shaping the timing of sex. Additionally, population densities can influence the evolution of the induction of sex and male production in two ways. First, density can act as a cue for time in the season when no direct cues are available. Second, in populations with density-dependent growth, the demographic cost of sex (forgoing direct reproduction) is reduced when recruitment of directly developing asexual offspring is hampered by high population density. In some cases, the best responses to uncertain cues lead to endogenously produced population cycles.

\section{Acknowledgments}

We are grateful to Dieter Ebert for stimulating discussions and to Russell Bonduriansky, Florence Débarre, Maurine Neiman, and an anonymous reviewer for their helpful comments. This project was funded by the Academy of Finland (Finnish Centre of Excellence in Biological Interactions Research) project SA-252411 (to H.K.) and the Swiss National Science Foundation.

\section{APPENDIX A}

Table A1: Model parameter definitions

\begin{tabular}{|c|c|}
\hline Model parameter & Definition \\
\hline$t$ & Breeding cycle within the season \\
\hline$T$ & $\begin{array}{l}\text { Breeding cycle when the season ends } \\
\text { reaching from } T_{\min } \text { to } T_{\max }\end{array}$ \\
\hline$p_{T}$ & $\begin{array}{l}\text { Probability that the season ends after } T \\
\text { breeding cycles }\end{array}$ \\
\hline$W$ & Fitness of a clone \\
\hline$G_{T}$ & $\begin{array}{l}\text { Gene copies that exist in ephippia at the end } \\
\text { of the season } T\end{array}$ \\
\hline$N_{t}$ & Population density at time $t$ \\
\hline$C$ & Individuals that mature from one clutch \\
\hline$s_{t}$ & Probability of reproducing sexually at time $t$ \\
\hline $\boldsymbol{s}_{t}^{r}$ & $\begin{array}{l}\text { Probability of resident females reproducing } \\
\text { sexually at time } t\end{array}$ \\
\hline$s_{t}^{\prime}$ & $\begin{array}{l}\text { Probability of mutant females reproducing } \\
\text { sexually at time } t\end{array}$ \\
\hline$m_{t}$ & $\begin{array}{l}\text { Conditional probability of producing males } \\
\text { if reproduction was not sexual }\end{array}$ \\
\hline$m_{t}^{r}$ & $\begin{array}{l}\text { Probability of resident females reproducing } \\
\text { sexually at time } t\end{array}$ \\
\hline$m_{t}^{\prime}$ & $\begin{array}{l}\text { Probability of mutant females reproducing } \\
\text { sexually at time } t\end{array}$ \\
\hline$d_{\mathrm{f}}$ & Mortality of mature females \\
\hline$d_{\mathrm{m}}$ & Mortality of mature males \\
\hline$F_{t}$ & $\begin{array}{l}\text { Number of mature females at breeding } \\
\text { cycle } t\end{array}$ \\
\hline$F_{t}^{r}$ & $\begin{array}{l}\text { Number of resident females at breeding } \\
\text { cycle } t\end{array}$ \\
\hline$F_{t}^{\prime}$ & $\begin{array}{l}\text { Number of mutant females at breeding } \\
\text { cycle } t\end{array}$ \\
\hline$M_{t}$ & Number of mature males at breeding cycle $t$ \\
\hline$M_{t}^{r}$ & $\begin{array}{l}\text { Number of resident males at breeding } \\
\text { cycle } t\end{array}$ \\
\hline$M_{t}^{\prime}$ & Number of mutant males at breeding cycle $t$ \\
\hline$g_{t}^{\prime}$ & Fitness gain of the mutant at time $t$ \\
\hline$p$ & $\begin{array}{l}\text { Probability that recombinant strategies can } \\
\text { copy the neighbor's strategy }\end{array}$ \\
\hline
\end{tabular}

\section{Literature Cited}

Alekseev, V., and W. Lampert. 2001. Maternal control of resting-egg production in Daphnia. Nature 414:899-901.

Altermatt, F., and D. Ebert. 2008. The influence of pool volume and summer desiccation on the production of the resting and dispersal stage in a Daphnia metapopulation. Oecologia 157:441-452.

Aparici, E., M. J. Carmona, and M. Serra. 1998. Sex allocation in haplodiploid cyclical parthenogens with density-dependent proportion of males. American Naturalist 152:652-657.

Bengtsson O., and A. Ceplitis. 2000. The balance between sexual and asexual reproduction in plants living in variable environments. Journal of Evolutionary Biology 13:415-422.

Berg, L. M., S. Palsson, and M. Lascoux. 2001. Fitness and sexual response to population density in Daphnia pulex. Freshwater Biology 46:667-677. doi:10.1046/j.1365-2427.2001.00704.x. 
Booksmythe, I., N. Gerber, D. Ebert, and H. Kokko. 2018. Daphnia females adjust sex allocation in response to current sex ratio and density. Ecology Letters 21:629-637.

Bradford, M. J., and D. A. Roff. 1993. Bet hedging and the diapause strategies of the cricket Allonemobius fasciatus. Ecology 74:1129-1135.

Burke, N. W., and R. Bonduriansky. 2017. Sexual conflict, facultative asexuality, and the true paradox of sex. Trends in Ecology and Evolution 32:646-652. doi:10.1016/j.tree.2017.06.002.

Burke, N. W., A. J. Crean, and R. Bonduriansky. 2015. The role of sexual conflict in the evolution of facultative parthenogenesis: a study on the spiny leaf stick insect. Animal Behaviour 101:117-127.

Caprioli, M., and C. Ricci. 2001. Recipes for successful anhydrobiosis in bdelloid rotifers. Hydrobiologia 446:13-17. doi:10.1023/A:10175566 02272.

Carmona, M. J., N. Dimas-Flores, E. M. Garca-Roger, and M. Serra. 2009. Selection of low investment in sex in a cyclically parthenogenetic rotifer. Journal of Evolutionary Biology 22:1975-1983.

Carvalho, G. R., and R. N. Hughes. 1983. The effect of food availability, female culture-density and photoperiod on ephippia production in Daphnia magna Straus (Crustacea: Cladocera). Freshwater Biology 13:37-46.

Cohen, D. 1966. Optimizing reproduction in a randomly varying environment. Journal of Theoretical Biology 12:119-129.

Decaestecker, E., L. De Meester, and J. Mergeay. 2009. Cyclical parthenogenesis in Daphnia: sexual versus asexual reproduction. Pages 295-316 in I. Schön, K. Martens, and P. Dijk, eds. Lost sex. Springer, Dordrecht, doi:10.1007/978-90-481-2770-2_15.

D'Souza, T. G., and N. K. Michiels. 2010. The costs and benefits of occasional sex: theoretical predictions and a case study. Iournal of Heredity 101:S34-S41.

Ebert, D. 2005. Ecology, epidemiology, and evolution of parasitism in Daphnia. National Library of Medicine, Bethesda, MD.

Ellner, S. 1985. ESS germination strategies in randomly varying environments. I. Logistic-type models. Theoretical Population Biology 28:5079.

Evans, M. E. K., and J. J. Dennehy. 2005. Germ banking: bet-hedging and variable release from egg and seed dormancy. Quarterly Review of Biology 80:431-451.

Furness, A. I., K. Lee, and D. N. Reznick. 2015. Adaptation in a variable environment: phenotypic plasticity and bet-hedging during egg diapause and hatching in an annual killifish. Evolution 69:14611475 .

Galimiov, Y., B. Walser, and C. R. Haag. 2011. Frequency and inheritance of non-male producing clones in Daphnia magna: evolution towards sex specialization in a cyclical parthenogen? Journal of Evolutionary Biology 24:1572-1583.

García-Roger, E. M., M. Serra, and M. J. Carmona. 2014. Bet-hedging in diapausing egg hatching of temporary rotifer populations: a review of models and new insights. International Review of Hydrobiology 99:96-106.

Gerber, N., I. Booksmythe, and H. Kokko. 2018b. Data from: Sex allocation theory for facultatively sexual organisms inhabiting seasonal environments: the importance of bet-hedging. American Naturalist Dryad Digital Repository, https://doi.org/10.5061/dryad.885r162.

Gerber, N., and H. Kokko. 2018. Abandoning the ship using sex, dispersal, or dormancy: multiple escape routes from challenging conditions. Philosophical Transactions of the Royal Society B. doi:10.1098 /rstb.2017.0424.

Gerber, N., H. Kokko, D. Ebert, and I. Booksmythe. 2018a. Daphnia invest in sexual reproduction when its relative costs are reduced.
Proceedings of the Roval Society B 285:20172176. doi:10.1098/rspb .2017 .2176 .

Gilbert, J. J., and T. Schröder. 2004. Rotifers from diapausing, fertilized eggs: unique features and emergence. Limnology and Oceanography 49:1341-1354.

Gourbière, S., and F. Menu. 2009. Adaptive dynamics of dormancy duration variability: evolutionary trade-off and priority effect lead to suboptimal adaptation. Evolution 63:1879-1892.

Graham, J. K., M. L. Smith, and A. M. Simons. 2014. Experimental evolution of bet hedging under manipulated environmental uncertainty in Neurospora crassa. Proceedings of the Roval Society B 281:20140706.

Green, R. F., and D. L. G. Noakes. 1995. Is a little bit of sex as good as a lot? \ournal of Theoretical Biology 174:87-96. doi:10.1006/jtbi .1995 .0081

Gremer, J. R., E. E. Crone, and P. Lesica. 2012. Are dormant plants hedging their bets? demographic consequences of prolonged dormancy in variable environments. American Naturalist 179:315-327.

Gremer, J. R., and D. L. Venable. 2014. Bet hedging in desert winter annual plants: optimal germination strategies in a variable environment. Ecology Letters 17:380-387.

Halkett, F., R. Harrington, M. Hullé, P. Kindlmann, F. Menu, C. Rispe, and M. Plantegenest. 2004. Dynamics of production of sexual forms in aphids: theoretical and experimental evidence for adaptive "coin-flipping" plasticity. American Naturalist 163:E112E125.

Hanski, I. 1988. Four kinds of extra long diapause in insects: a review of theory and observations. Annales Zoologici Fennici 25:3753.

Hebert, PDN. 1981. Obligate asexuality in Daphnia. American Naturalist 117:784-789. doi:10.1086/283761

Innes, D. 1997. Sexual reproduction of Daphnia pulex in a temporary habitat. Oecologia 111:53-60.

Innes, D., C. J. Fox, and G. L. Winsor. 2000. Avoiding the cost of males in obligately asexual Daphnia pulex (Leydig). Proceedings of the Roval Society B 267:991-997. doi:10.1098/rspb.2000.1101.

Kahn, A. T., M. D. Jennions, and H. Kokko. 2015. Sex allocation, juvenile mortality, and the costs imposed by offspring on parents and siblings. Journal of Evolutionary Biology 28:428-437.

Kahn, A. T., H. Kokko, and M. D. Jennions. 2013. Adaptive sex allocation in anticipation of changes in offspring mating opportunities. Nature Communications 4:1603.

Kleiven, O. T., P. Larsson, and A. Hobaek. 1992. Sexual reproduction in Daphnia magna requires three stimuli. Oikos 65:197-206.

Lehtonen, J., M. D. Jennions, and H. Kokko. 2012. The many costs of sex. Trends in Ecology and Evolution 27:172-178. doi:10.1016 /j.tree.2011.09.016.

Li, X.-Y., J. Lehtonen, and H. Kokko. 2017. Sexual reproduction as bethedging. Pages 217-234 in J. Apaloo and B. Viscolani, eds. Advances in dynamic mean field games: theory, applications, and numerical methods. Annals of the International Society of Dynamic Games. Springer, Basel.

McNamara, J. M., S. R. X. Dall, P. Hammerstein, and O. Leimar. 2016. Detection vs. selection: integration of genetic, epigenetic and environmental cues in fluctuating environments. Ecology Letters 19:1267-1276.

McNamara, J. M., and A. I. Houston. 2009. Integrating function and mechanism. Trends in Ecology and Evolution 24:670-675.

McNamara, J. M., J. N. Webb, E. J. Collins, T. Székely, and A. I. Houston. 1997. A general technique for computing evolutionarily stable strate- 
gies based on errors in decision-making. Lournal of Theoretical Biology 189:211-225.

Pinceel, T., B. Vanschoenwinkel, W. Hawinkel, K. Tuytens, and L. Brendonck. 2017. Aridity promotes bet hedging via delayed hatching: a case study with two temporary pond crustaceans along a latitudinal gradient. Oecologia 184:161-170.

Roulin, A. C., J. Routtu, M. D. Hall, T. Janicke, I. Colson, C. R. Haag, and D. Ebert. 2013. Local adaptation of sex induction in a facultative sexual crustacean: insights from QTL mapping and natural populations of Daphnia magna. Molecular Ecology 22:3567-3579.

Schierwater, B., and C. Hauenschild. 1990. A photoperiod determined life-cycle in an oligochaete worm. Biological Bulletin 178:111-117.

Schröder, T. 2005. Diapause in monogonont rotifers. Hydrobiologia 546:291-306. doi:10.1007/1-4020-4408-9_30.

Serra, M., E. Aparici, and M. J. Carmona. 2008. When to be sexual: sex allocation theory and population density-dependent induction of sex in cyclical parthenogens. Journal of Plankton Research 30:12071214.

Simon, J.-C., C. Rispe, and P. Sunnucks. 2002. Ecology and evolution of sex in aphids. Trends in Ecology and Evolution 17:34-39.

Slatkin, M. 1974. Hedging one's evolutionary bets. Nature 250:704-705. Spencer, M., N. Colegrave, and S. S. Schwartz. 2001. Hatching fraction and timing of resting stage production in seasonal environments: effects of density dependence and uncertain season length. Journal of Evolutionary Biology 14:357-367. doi:10.1046/j.1420 -9101.2001.00297.x.

Starrfelt, J., and H. Kokko. 2012. Bet-hedging: a triple trade-off between means, variances and correlations. Biological Reviews 87:742-755.
Stelzer, C. P. 2012. Population regulation in sexual and asexual rotifers: an eco-evolutionary feedback to population size? Functional Ecology 26:180-188. doi:10.1111/j.1365-2435.2011.01918.x.

Stelzer, C. P., and J. Lehtonen. 2016. Diapause and maintenance of facultative sexual reproductive strategies. Philosophical Transactions of the Roval Societv B 371:20150536.

Stelzer, C. P., and T. W. Snell. 2003. Induction of sexual reproduction in Brachionus plicatilis (Monogononta, Rotifera) by a densitydependent chemical cue. Limnology and Oceanography 48:939943.

Tarazona, E., E. M. Garcia-Roger, and M. J. Carmona. 2017. Experimental evolution of bet hedging in rotifer diapause traits as a response to environmental unpredictability. Oikos 126:1162-1172.

Tessier, A. J., and C. E. Caceres. 2004. Differentiation in sex investment by clones and populations of Daphnia. Ecology Letters 7:695-703.

Tielbörger, K., M. Petruů, and C. Lampei. 2012. Bet-hedging germination in annual plants: a sound empirical test of the theoretical foundations. Oikos 121:1860-1868.

Via, S., and R. Lande. 1985. Genotype-environment interaction and the evolution of phenotypic plasticity. Evolution 39:505-522.

West, S. 2009. Sex allocation. Princeton University Press, Princeton, NJ.

Wittmann, M. J., M. A. Lewis, J. D. Young, and N. D. Yan. 2011. Temperature-dependent Allee effects in a stage-structured model for Bythotrephes establishment. Biological Invasions 13:2477-2497.

Associate Editor: Russell Bonduriansky Editor: Alice A. Winn

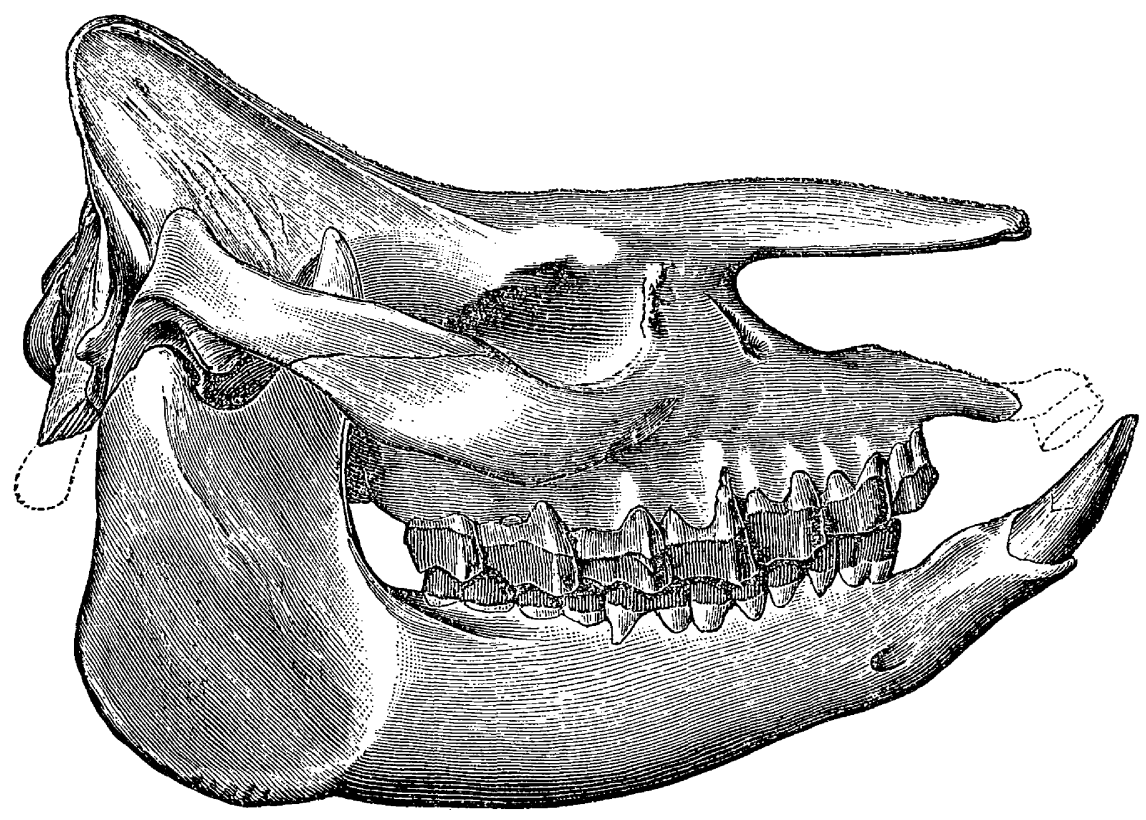

"Twelve species of mammals which may be called rhinoceroses, have been defined from materials obtained from the Tertiary formations of North America; and five additional species have been distinguished, which may be regarded as more or less nearly allied to that family. . . . The family still exists in Asia and Africa, but in Europe it disappeared during the glacial epoch. In North America it became extinct at a still earlier period, no remains of rhinoceroses having been found in beds of later age than the Loup Fork, or Upper Miocene period." From "On the Extinct American Rhinoceroses and their Allies" by E. D. Cope (The American Naturalist, 1879, 13:771a-771j). 